\author{
A TWO-DIMENSIONAL READOUT DRIFT CHAMBER \\ WITH PRINTED CIRCUIT DELAY LINES \\ M. Atac, R. Bosshard ${ }^{\dagger}, Y$. Kang \\ Fermi National Accelerator Laboratory \\ P. O. BOX 500 \\ Batavia, Illinois 60510 \\ tLaboratory Accelerateur Lineaire, Orsay, France
}

December, 1975

\begin{abstract}
A two-dimensional readout drift chamber with printed circuit zig-zag delay lines has been developed. Track position accuracies of 3-5 mm from the delay line and 80-100 from the electron drift time have been obtained.
\end{abstract}




\section{A TWO-DIMENSIONAL READOUT DRIFT CHAMBER WITH PRINTED CIRCUIT DELAY LINES}

\section{Introduction}

Obtaining two-dimensional coordinate measurements of a track from a proportional or a drift chamber cell is desirable especially for high multiplicity experiments. Having a pair of correlated coordinates for every track would reduce multitrack ambiguities and consequently decrease computing costs. Here we offer an elegant and practical delay line readout which can be conveniently constructed in a parallel foil drift chamber (PFDC). ${ }^{1,2}$ In this case, printed circuit tape delay lines (zig-zag lines) replacing the parallel arift foil electrodes provide a second coordinate for a track in a cell. The second coordinate is measured through the zig-zag delay lines by induced pulses. On the other hand, the first coordinate is obtained from the drift time of the electrons produced by ionization during the passage of a charged particle with an accuracy of 80-100 ${ }^{i}, 2$ This type of delay line readout eases the difficulties in measuring a longitudinal track coordinate in a cylindrical geometry.

In the following, we will describe a prototype PFDC of $50 \mathrm{~cm} \times 50 \mathrm{~cm}$ active area $(25 \mathrm{cel} 1 \mathrm{~s})$ in which two of the aluminum foils were replaced with a pair of printed circuit thin foil delay lines. The results show that a track position accuracy $\sigma$ of 3 to $5 \mathrm{~mm}$ can be obtained along the delay line. 


\section{Chamber Construction}

Figure 1 shows a simplified perspective view of the chamber together with a cross sectional view. In the perspective view, the dimensions shown are not to scale for clarity and simplicity.

The dimensions of the cells are indicated in the cross sectional view. The chamber has a sensitive area of $50 \mathrm{~cm} \times 50 \mathrm{~cm}$ and it contains 25 drift cells. The signal wires were soldered in positions within $5 \mu$ accuracy and the delay lines were glued with Eastman 910 to the positioning blocks within $25 \mu$ accuracy. We will not give every detail of PFDC construction since some of the details are given in the earlier reports.

\section{The Delay Lines}

The delay line has two characteristic features. It provides a uniform electric field and it senses the induced signal. Consequently, results in delay time measurements, at its ends from which the track positions along the sense wire, are obtained. Desirable characteristics of such a delay line are:

a) High characteristic impedance $z$ to achieve primarily good signal to noise ratio which is proportional to $\mathrm{z}^{\frac{1}{2}}$.

b) Good coupling efficiency $n$ (see page 3).

c) Long delay time, $\tau$, per unit length.

d) A reasonable balance among signal losses due to d.c. resistance, skin effect, dispersion and the thickness of the conductive material (multiple coulomb scattering). Most of the above objectives are achieved by maintaining a flat geometry by using a printed circuit technique. This is 
illustrated in Figure 2a. The delay lines are photo-etched on

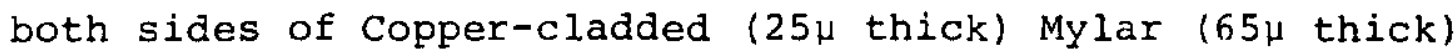
strips. The rest of the dimensions are given with the figure. The conductive planes on a strip are shifted by a half period in order to boost the inductance $\mathrm{L}$ while maintaining a constant capacitance $C$, thus simultaneously increasing impedance $Z$ and the delay time $\tau$.

Figure $2 \mathrm{~b}$ shows how this takes place. The currents $I$ in the two conductors (in the region where they overlap) are in the same direction thereby enhancing the mutual inductance. From theory, the inductance of an iterative loop can be seen to be four times the inductance of a single loop. For the lines considered here, the single loop inductance is three times the inductance of the strip (non-shifted zig-zag) of the same length. As a result, we get a factor of 12 in inductance, thus an increase of about a factor of 3.5 in $z$ and $\tau$. Some details on the delay lines are given in Reference 3 .

The coupling efficiency $n$ of the delay line looking toward the anode wire is related by the difference between the induced charge on the forward zig-zag plane $q_{f}$ and that seen by the backward zig-zag plane $\mathrm{q}_{\mathrm{b}}$. Since these charges are proportional to the capacitances $c_{f}$ and $c_{b}$, we define $n$ to be:

$$
\eta=\frac{c_{f}-c_{b}}{c_{f}+c_{b}}
$$

The $\eta$ is also dependent on the ratio of the width $w$ over the spacing $S$ of the delay line. To improve $n$ the ratio $W / S$ of the 
forward plane can be increased relative to that of the backward plane. In this way, coupling efficiency of $80 \%$ has been achieved without losing much of the properties of the line.

The skin effect and the d.c. resistance are mainly the limiting factors in choosing a practical length for a delay line. To see the characteristic skin effect, we shall take the value of the rise time for a step function to rise to its $50 \%$ amplitude as given by the thesry of long cables (Reference 5),

$$
t_{50}=\frac{\rho \mu}{4 z^{2}}\left(\frac{l_{0}}{w}\right)^{2}
$$

where $\rho$ is the resistivity, $\mu$ is the permeability, $z$ is the characteristic impedance, $W$ is the width, and the $\ell_{0}$ is the total length of the conductor in one plane. Four times this value is taken in the case of the delay line. For copper, we obtain the following practical formula in MKS units:

$$
t_{50} \simeq \frac{2.10^{-4}}{z^{2}}\left(\frac{\ell}{w}\right)^{2}
$$

We see from this expression that it is important to achieve a high impedance $z$ for our $50 \mathrm{~cm}$ long delay line. We get $t_{50} \simeq 3.6 \mathrm{nsec}$ from the conductor of $250 \mu$ width and $l_{0} \simeq 8 \mathrm{~m}$ with an impedance of $75 \Omega$ and with a pulse attenuation factor of $20 \%$.

\section{Tests with $\mathrm{X}$-Ray Source}

Well-collimated $5.9 \mathrm{keV}$ x-rays of $\mathrm{Fe}^{55}$ were used for the tests to measure some of the characteristics of the chamber and the delay Iines, while $\mathrm{Ar} / \mathrm{C}_{2} \mathrm{H}_{2} / \mathrm{CH}_{4}$ gas mixture is flowed through 
the chamber. The gas is flow mixed with flow ratios of $22 \% \mathrm{C}_{2} \mathrm{H}_{2}$ and $78 \%$ of $\mathrm{Ar}-\mathrm{CH}_{4}$ (90\% Ar - $10 \% \mathrm{CH}_{4}$ pre-mixed). A gas gain of $5 \times 10^{5}$ is achieved with this gas at a voltage of $2.3 \mathrm{kV}$ applied to the chamber cells operating in the proportional region. Figure 3 shows induced signals which are integrated over 5 usec obtained from the delay Iine with external triggers from the anode pulses. The $5.9 \mathrm{keV}$ x-ray line and the escape line are clearly seen in the figure. An inverting charge amplifier is used to obtain the picture. The induced signal on the line is about $20 \%$ s. the anode signal. Figure 4 shows the current pulses which are obtained differentially from the delay lines using the current amplifier shown in Figure 5 . Since the pulses are quite small, the success of the delay line operation depends on the signal to noise ratio. Care is taken to reduce the noise with this amplifier design. These current pulses are about $6 \%$ of the full current signals obtained from the anode wire.

The collimated source was moved in steps of $2 \mathrm{~cm}$ along the drift chamber cell. Figure 6 shows some delay time spectra obtained from the difference time measurements which are measured by a time-to-amplitude converter and stored in a pulse height analyzer. Figure 7 shows a good linear relation between the position of the source and the mean delay time difference. When the source is displaced $1 \mathrm{~cm}$ in the difference measurement this corresponds to $4.4 \mathrm{nsec}$, thus providing $2.2 \mathrm{nsec} / \mathrm{cm}$ delay in the transmission of the induced pulses in both directions of the delay line. The standard deviations from the mean values in measurements of the source positions along the line are determined 
to be $\sigma=3 \mathrm{~mm}$ in the entire range shown in the figure.

Tests at the Beam Line

Further tests were carried out at the M6 West beam line of the Meson Laboratory using $100 \mathrm{GeV} / \mathrm{c}$ pion beam. The beam was defined by a pair of plastic scintillators of $5 \mathrm{~mm} \times 10 \mathrm{~mm}$ size $(5 \mathrm{~mm}$ width is in the direction of the delay line). The delay times were measured by a pair of time digitizer circuits in CAMAC system as the chamber moved relative to the beam. The trigger pulse obtained from the anode wire started the TDC's. The data was stored in tapes of a PDP-1.1 computer and analyzed off-line. Figure 8 shows two typical superimposed current pulses obtained from one end of the delay line. The time structure is due to successive gas multiplication of electrons as they reach the anode wire. This geometrical effect is one of the limiting factors of multiple track resolution of a drift chamber cell. The width of the time structure is varied between 100 to $150 \mathrm{nsec}$ for our geometry. Figure 9 shows a good linear relation between the track position and the delay time. A position accuracy of $\sigma=5 \mathrm{~mm}$ was obtained in the entire range.

The delay line system efficiency for obtaining a signal above the noise level was measured to be about $60 \%$ for the pion beam. We believe that this can be improved with an asymmetric construction of the chamber as indicated in Figure 10 by increasing the capacitance between the signal wires and the delay 1 ine. 


\section{REFERENCES}

1. M. Atac and W. E. Taylor, Nucl. Instr. and Meth. 120 (1974), 147-151.

2. M. Atac and C. Ankenbrandt, IEEE Transactions on Nuclear Science, Vol. NS-22, No. I (1975), 306.

3. R. Bosshard, R. L. Chase, J. Fisher, and V. Radeka, Brookhaven National Laboratory preprint.

4. G. Amsel and R. Bosshard, Rev. of Scientific Instr., Vol. 41, No. $4(1970), 503-514$.

5. R. I. Wigington and N. S. Nahman, PIRE 45 (1957), 166. 


\section{FIGURE CAPTIONS}

Figure 1.. A simplified perspective view and a cross section view of the chamber.

Figure 2. a) A descriptive view of the delay line strip.

b) Schematic view of the current loops.

Figure 3. Induced pulses at one end of the delay line obtained from an $\mathrm{Fe}^{55}$ source. The $5.9 \mathrm{keV} \mathrm{x}$-ray line and the escape line are seen in the picture. Pulses are integrated over $5 \mu \mathrm{sec}$.

Figure 4. The current pulses obtained from the delay line using $\mathrm{Fe}^{55}$ source and the amplifier shown in Figure 5. The scales are $20 \mathrm{nsec}$ per division and $0.1 \mathrm{v}$ per division.

Figure 5. The circuit diagram of the current amplifier.

Figure 6. Delay time spectra obtained from the pulse height analyzer using a time-to-amplitude converter. The $\mathrm{Fe}^{55}$ source is moved in steps of $2 \mathrm{~cm}$ along the delay line.

Figure 7. Source position to delay time relation.

Figure 8. A picture of current pulse time structure resulted from $100 \mathrm{GeV} / \mathrm{c}$ pion beam tracks. Horizontal scale is 50 nsec per division.

Figure 9. Track position to delay time relation.

Figure 10. A schematic view of the asymmetric construction of the drift chamber to improve the coupling efficiency of the delay line. 
CROSS-SECTIONAL VIEW

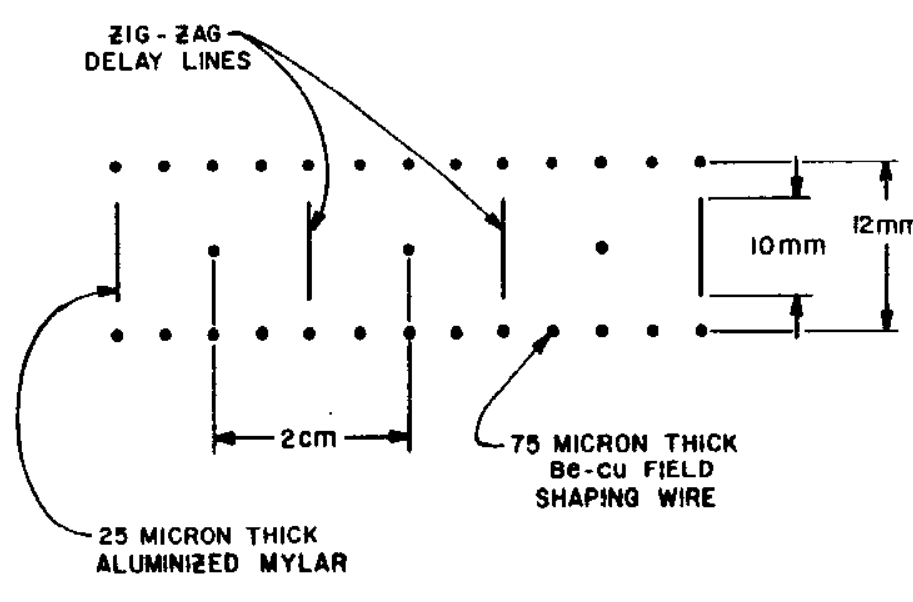

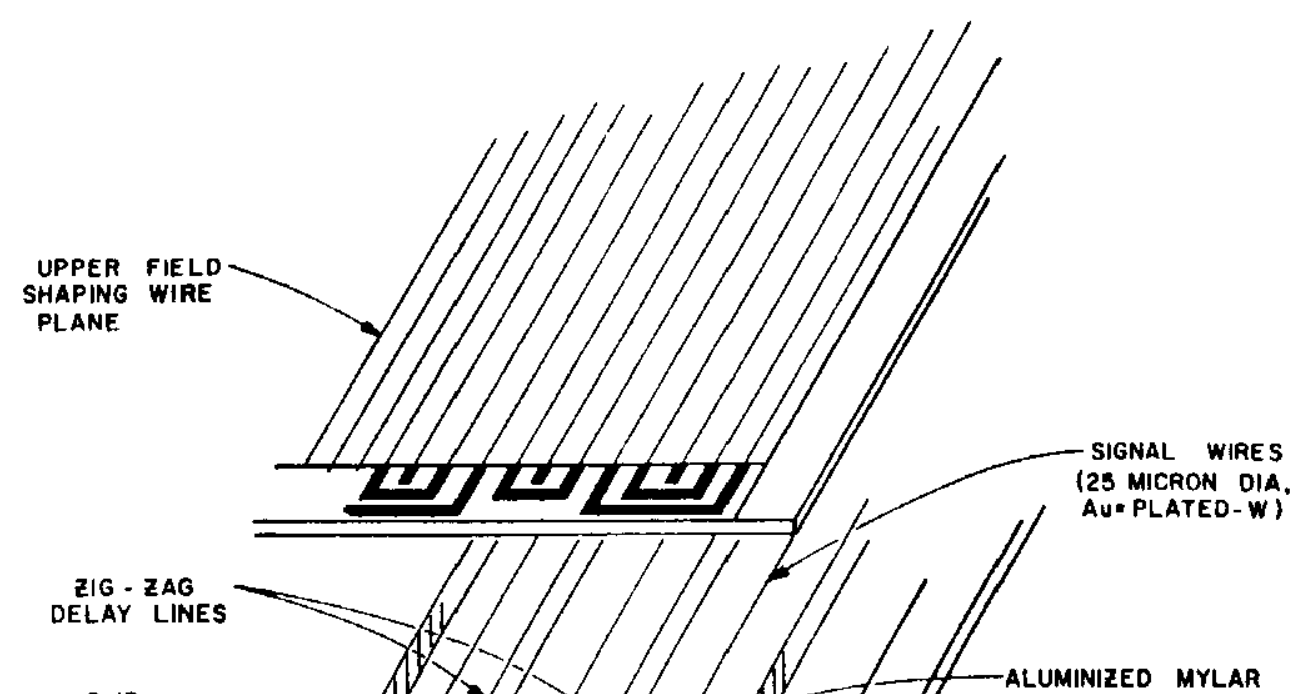

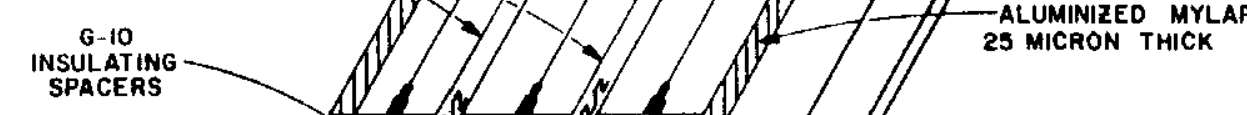

PERSPECTIVE VIEW

FIG. I 


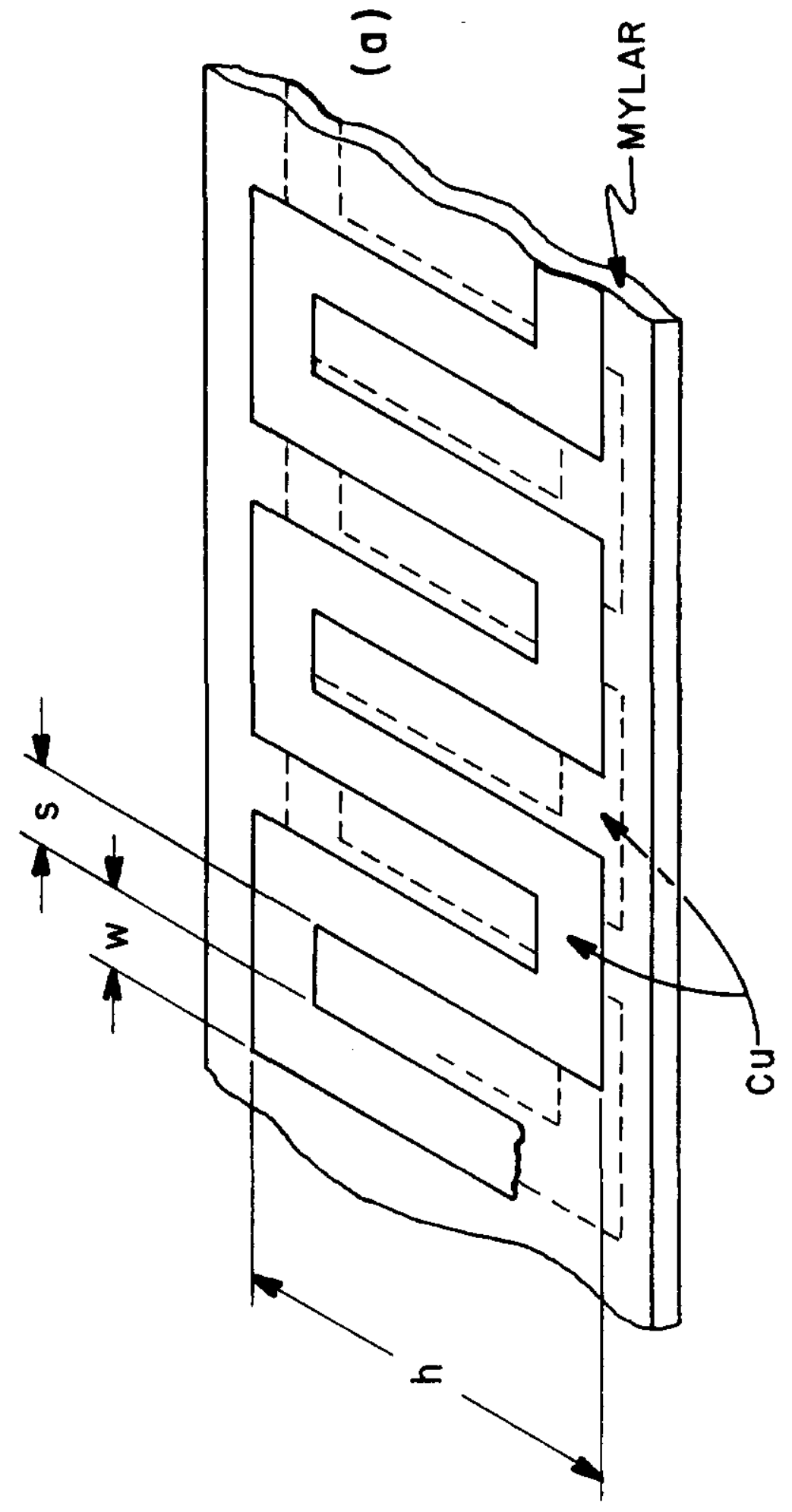

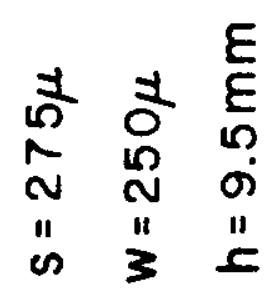

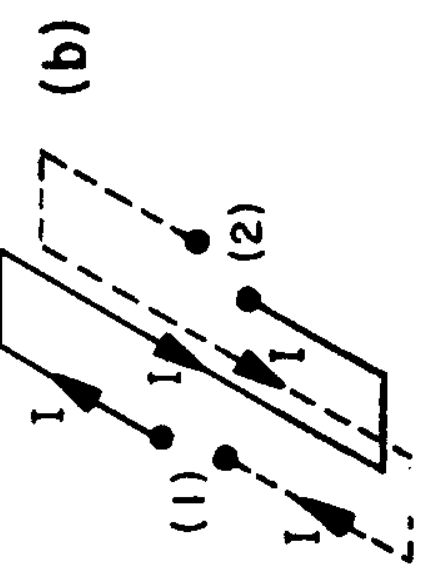




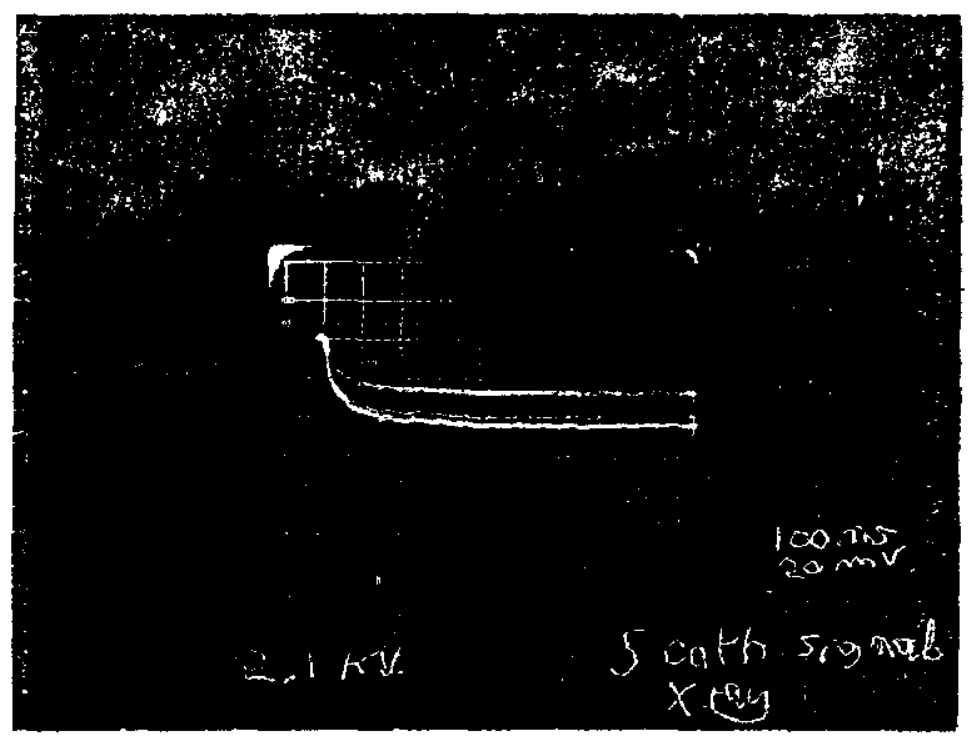

FIG. 3

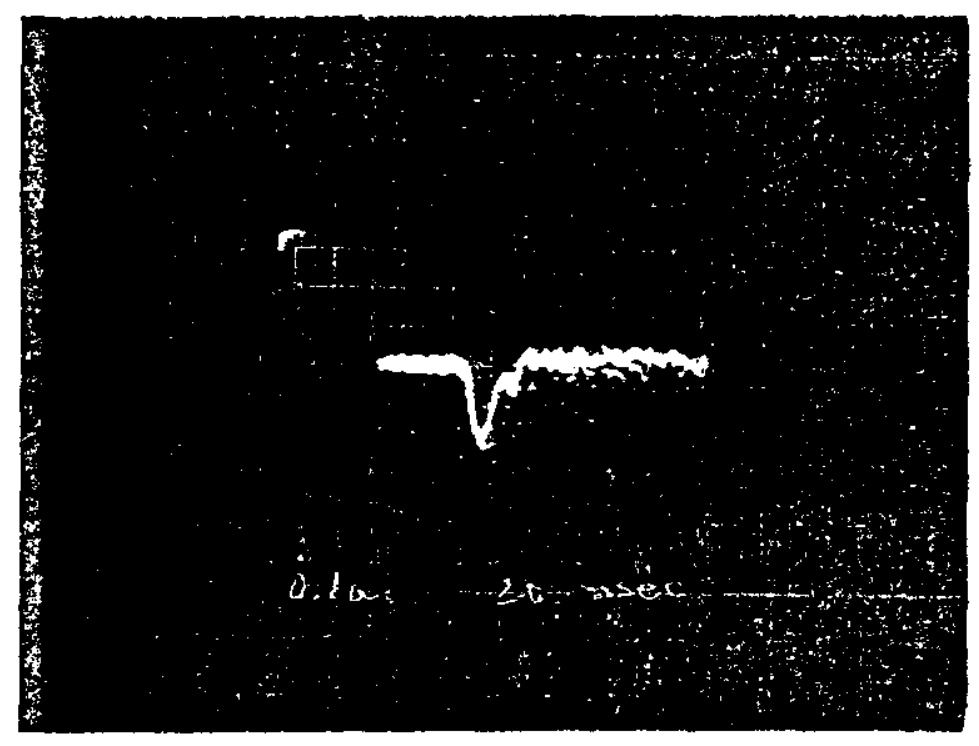

FIG. 4 


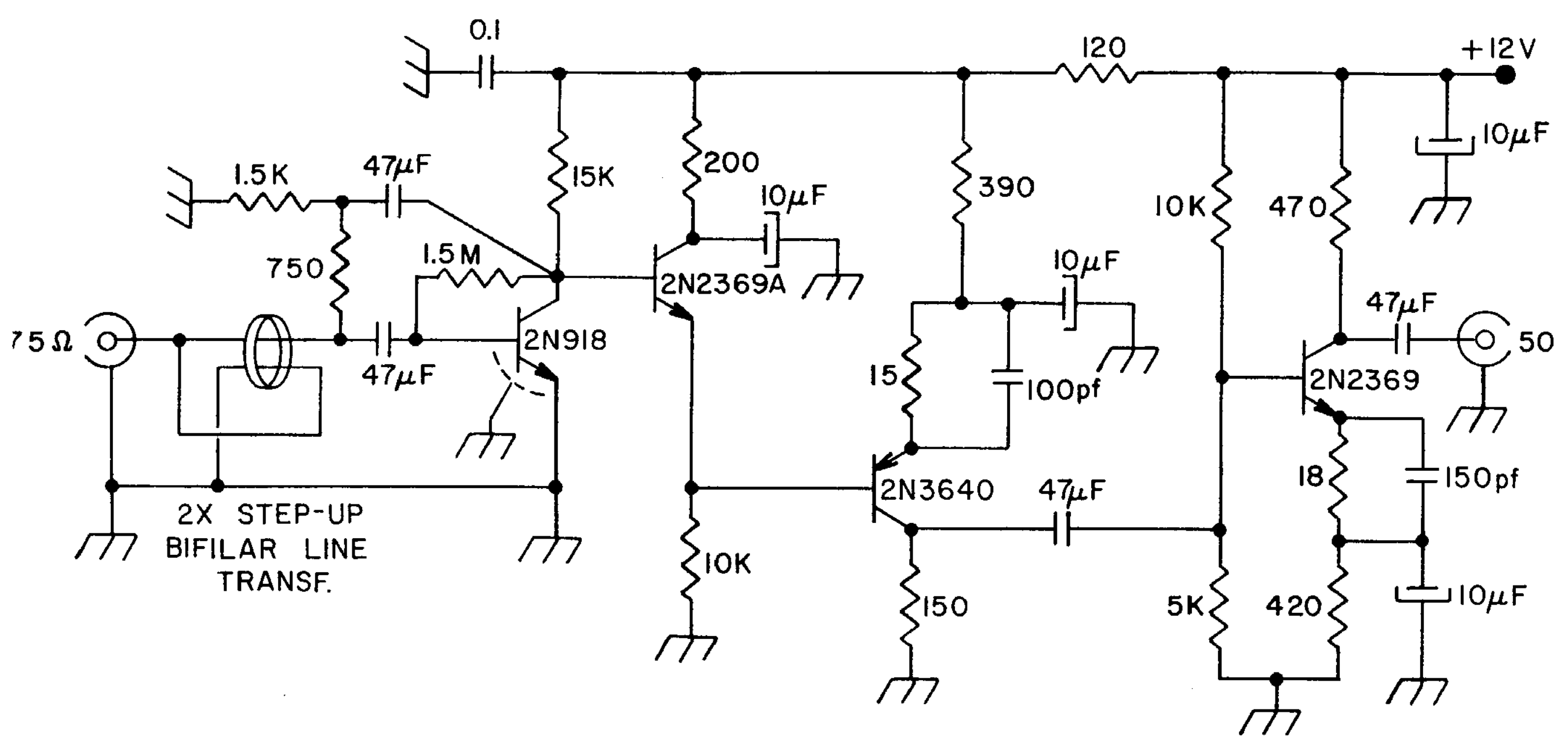

AMPLIFIER CIRCUIT

FIG. 5 
FN-286

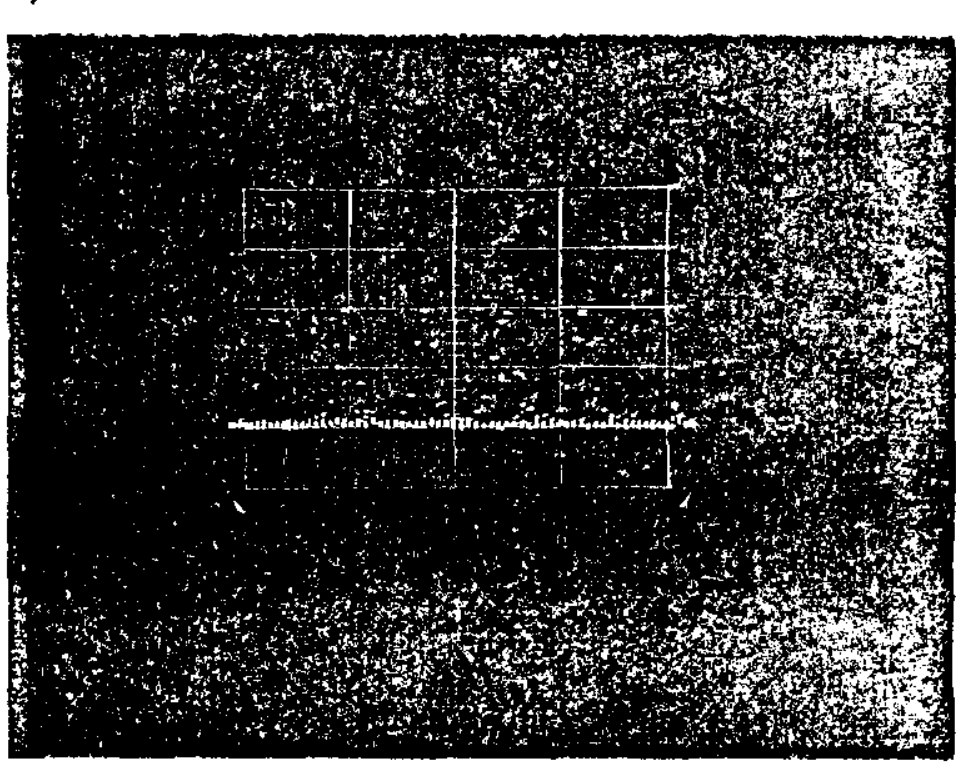

FIG. 6 
FN-286

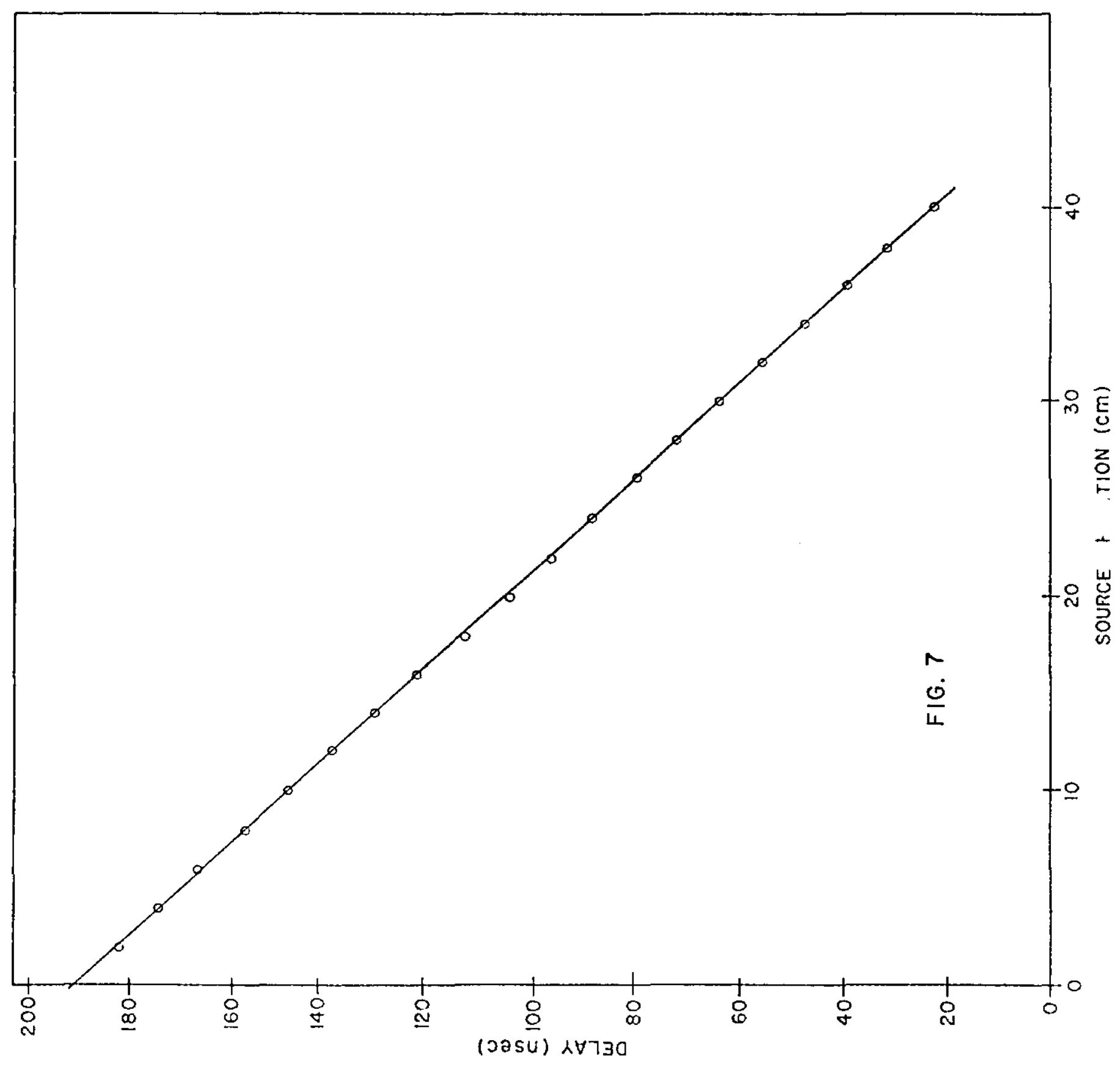


FN-286

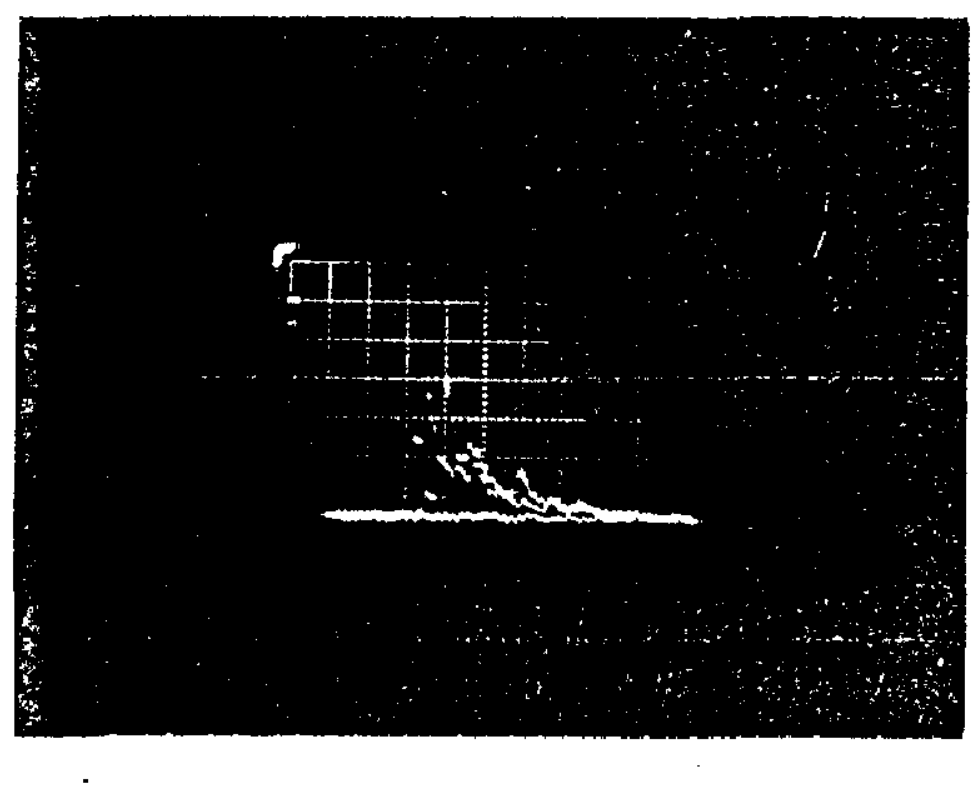

FIG. 8 


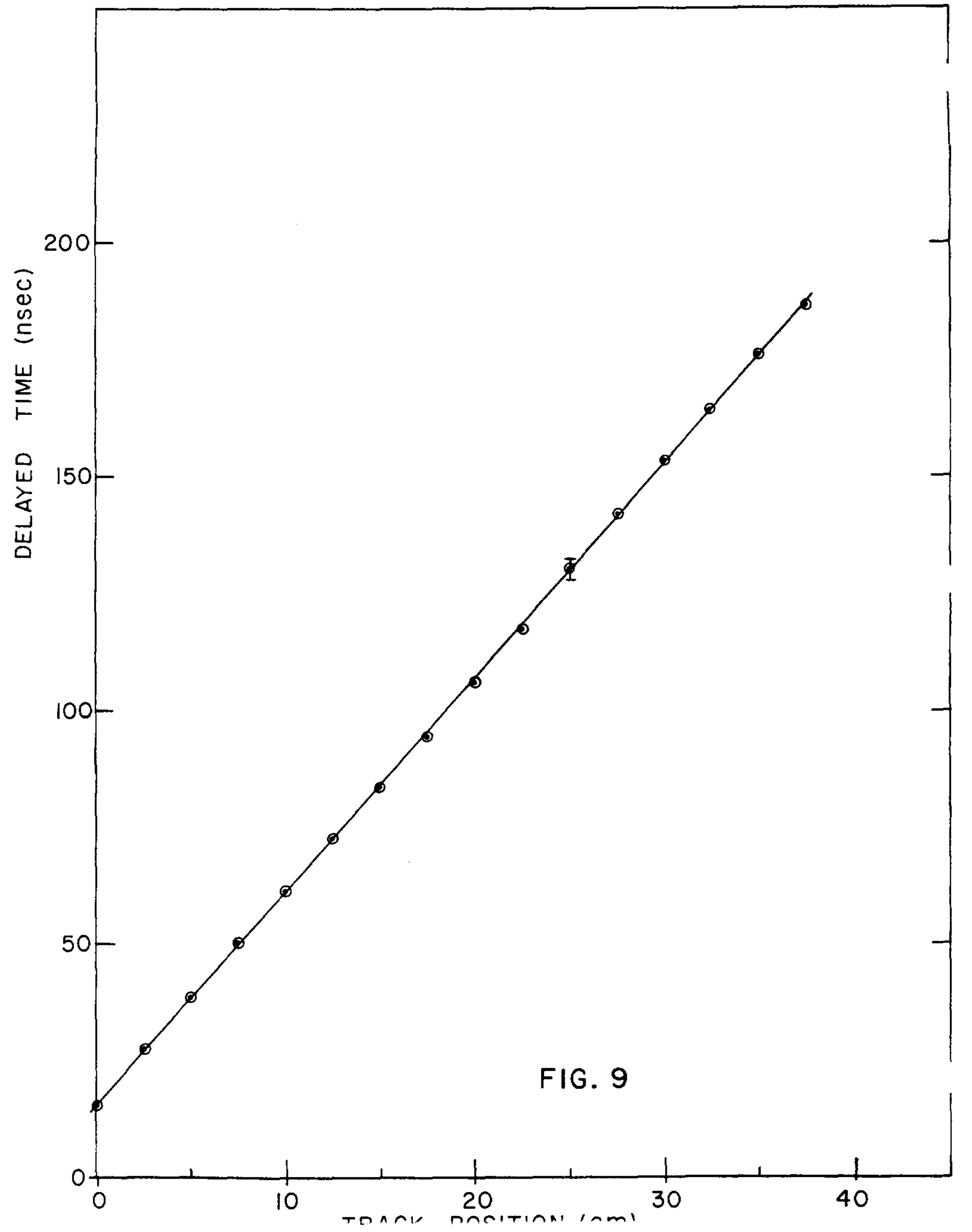




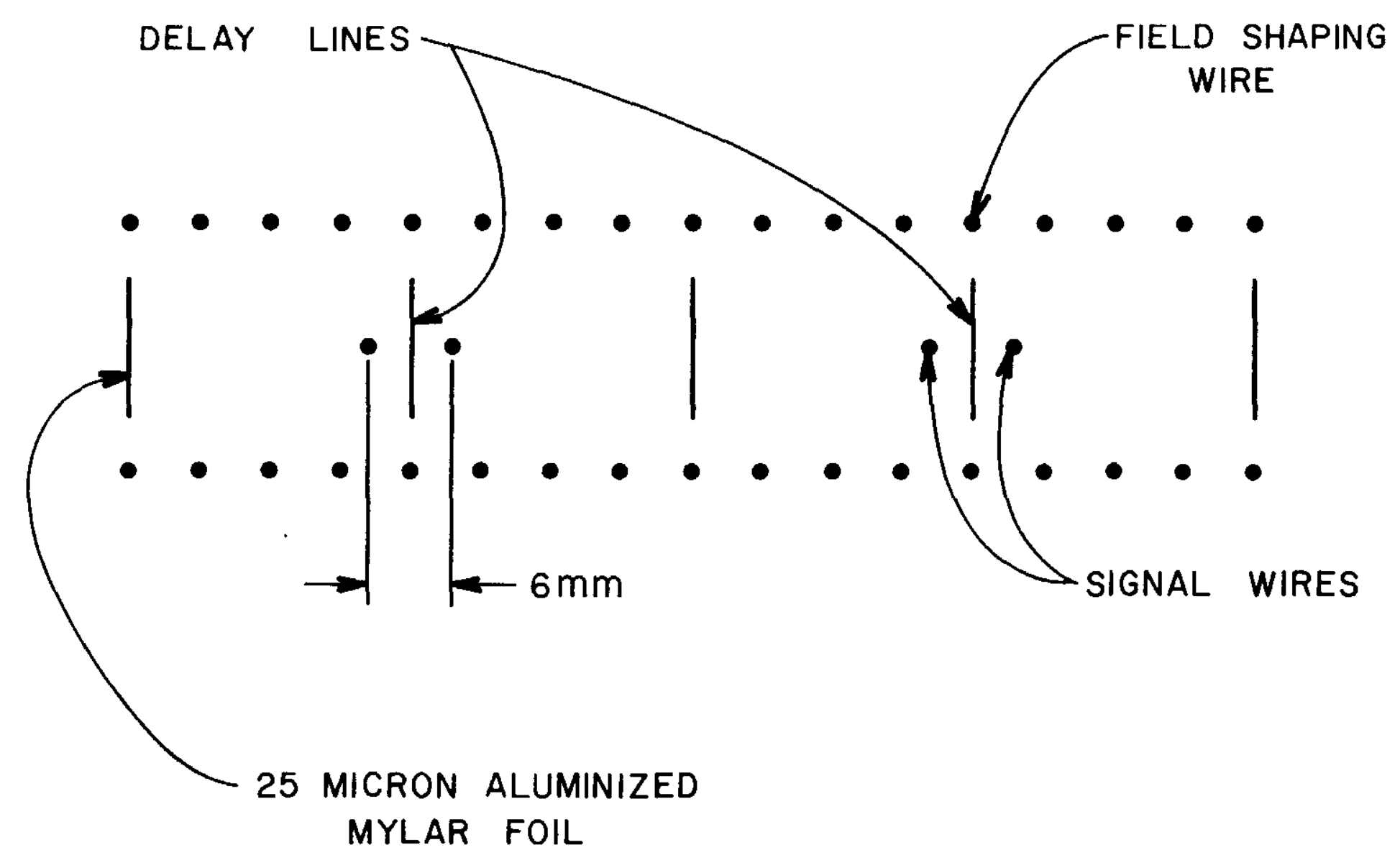

FIG. 10 